\title{
Consideraciones metodológicas y éticas para la conducción de ensayos clínicos controlados aleatorizados (ECCA) sobre intervenciones para las adicciones
}

\author{
Rodrigo Marín-Navarrete, ' José Fernández-Mondragón, ' Angélica Eliosa-Hernández,' \\ Liliana Templos Nuñez,' Javier Graue-Moreno,' Diana Galván-Sosa, ' Carla Fernández-De la Fuente,' \\ Georgina Gómez-Valencia, ${ }^{1}$ Carlos Berlanga ${ }^{2}$
}

Artículo original

\section{SUMMARY}

According to epidemiologic reports, the harmful use of alcohol and illicit drugs has increased among the Mexican population. This use is associated to several risks and issues that affect public health and the public well-being of the country. In this article it is acknowledged the need for development of treatment models and interventions whose therapeutic value is demonstrated by scientific evidence, that respond to the attention needs of the population affected by substance use in our country and that can be generalized in community clinical practice.

In clinical research, randomized controlled clinical trials (RCT's) are the "gold standard" to demonstrate the effect of a therapeutic intervention. A randomized controlled clinical trial is a prospective study in which the effect, value and safety of one or various experimental interventions are tested against a "control" intervention in human subjects.

Acknowledging that in Mexico there is a lack of research on addiction treatment that is compliant with all the requirements to be considered as an RCT, this article presents some methodological and ethical considerations that are neccesary for their design and conduction. These considerations include from the establishment of a relevant research question and objectives, adequate study design, development of strategies for data management, statistical analysis, monitoring of interventions, safety monitoring and research quality assurance and protection of human subjects.

Key words: Substance use disorders, treatment, controlled clinical trials, randomized clinical trials, methodology, ethical aspects.

\section{RESUMEN}

Según reportes epidemiológicos, el consumo nocivo de alcohol y drogas ilegales dentro de la población mexicana ha ido en aumento, lo que se asocia a varios riesgos o problemáticas que afectan la salud y bienestar públicos del país. Se reconoce la necesidad de desarrollar modelos de tratamiento e intervenciones cuyo valor terapéutico esté respaldado por la evidencia científica, que respondan a las necesidades de atención de la población afectada por el consumo de sustancias en nuestro país y que puedan generalizarse en la práctica clínica comunitaria.

Dentro de la investigación clínica, el "estándar de oro" para demostrar el efecto de una intervención terapéutica son los ensayos clínicos controlados aleatorizados (ECCA). Un ECCA es un estudio prospectivo en el cual se prueba el efecto, valor y seguridad de una o varias intervenciones experimentales contra una intervención "control" en sujetos humanos.

Reconociendo que en México hay una falta de investigaciones sobre tratamientos para las adicciones que cumplan con todos los requisitos para ser considerados ECCA, en este artículo se presentan distintas consideraciones metodológicas y éticas que deben tomarse en cuenta para su diseño y conducción en la materia; abarcando aspectos que parten desde el establecimiento de una pregunta y objetivos relevantes hasta el diseño adecuado del estudio y el desarrollo de estrategias para la administración de datos, análisis estadístico, monitoreo de las intervenciones, monitoreo de seguridad y aseguramiento de la calidad de la investigación y protección de los sujetos humanos que participan.

Palabras clave: Trastornos por consumo de sustancias, tratamiento, ensayos clínicos controlados, ensayos clínicos aleatorizados, metodología, aspectos éticos. 


\section{INTRODUCCIÓN}

Diversos reportes internacionales señalan que existe una tendencia hacia el aumento, o la estabilización, en la prevalencia del consumo nocivo de alcohol y drogas en la población mundial y nacional. ${ }^{1,2}$ Dicho consumo se encuentra asociado a diversos riesgos y problemas de impacto y relevancia para la salud y el bienestar público como la comorbilidad psiquiátrica, la mortalidad por enfermedades crónicas, los accidentes automovilísticos que causan muertes y discapacidad, entre otras más que generan un deterioro en la calidad de vida y estabilidad de la población. ${ }^{3-7}$ Ante este escenario es de vital importancia que las intervenciones dedicadas a minimizar el impacto del consumo de sustancias se encuentren razonablemente fundamentadas por los hallazgos de la investigación clínica. ${ }^{6}$

Entre los principios recomendados para el tratamiento del consumo de sustancias por la Organización Mundial de la Salud (OMS) y por la Oficina de las Naciones Unidas para la Droga y el Delito (UNODC) se encuentra el desarrollo y diseminación de modelos de tratamiento basados en evidencia científica que sean reproducibles y sensibles a las distintas necesidades de cada paciente y de su contexto socio-cultural. ${ }^{8}$ Dentro de las investigaciones clínicas, el "estándar de oro" para evaluar el efecto de una intervención es el modelo de ensayos clínicos controlados aleatorizados (ECCA). Un ensayo clínico es un estudio prospectivo en humanos en el cual se compara el efecto, valor y/o seguridad de una o más intervenciones terapéuticas contra una condición "estándar", que semeje a un experimento por control para establecer relaciones causa-efecto.,10 Para que un ensayo clínico pueda cumplir su objetivo debe ser diseñado y conducido de acuerdo a principios científicos sólidos y debe ser reportado de manera apropiada. ${ }^{11,12}$ Diversos esfuerzos realizados en otros países han buscado conducir ensayos clínicos con rigor científico para evaluar intervenciones especializadas en el tratamiento de las adicciones con relativo éxito, llevando a la diseminación de modelos de atención basados en evidencia a escenarios comunitarios de atención. ${ }^{13}$ Reconociendo que en México hace falta conducir investigaciones clínicas sobre tratamientos para las adicciones que cumplan con los estándares y rigor científico de un ECCA, ${ }^{14}$ este artículo tiene por objetivo presentar algunas consideraciones metodológicas y éticas que deben tenerse en mente para su diseño y conducción.

\section{CONSIDERACIONES METODOLÓGICAS}

\section{De acuerdo al tipo de estudio}

El establecer un diseño adecuado en la investigación de tratamientos en adicciones implica una mayor complejidad debido a la heterogeneidad de los pacientes consumidores de sustancias ${ }^{2,8}$ y a que no todos los tratamientos funcionan de la misma manera. ${ }^{15-17}$ También se deben tomar en cuenta otras variables relacionadas con la investigación de tratamientos y con la práctica clínica en adicciones. Dichas variables se pueden conceptualizar de la siguiente manera: ¿en cuál población?, ¿cuál intervención terapéutica (en qué dosis, a lo largo de cuánto tiempo)?, ¿aplicada por qué profesionales, en qué escenarios, con cuáles resultados se cree que está ligada la intervención y cuáles son los beneficios o ventajas que representa con respecto a lo ya disponible? ${ }^{9,18}$ Así, los ECCA pueden clasificarse a partir de varios criterios distintos: por tipo de tratamiento, por diseño, por objetivo y por fase o etapa de desarrollo del tratamiento (figura 1).
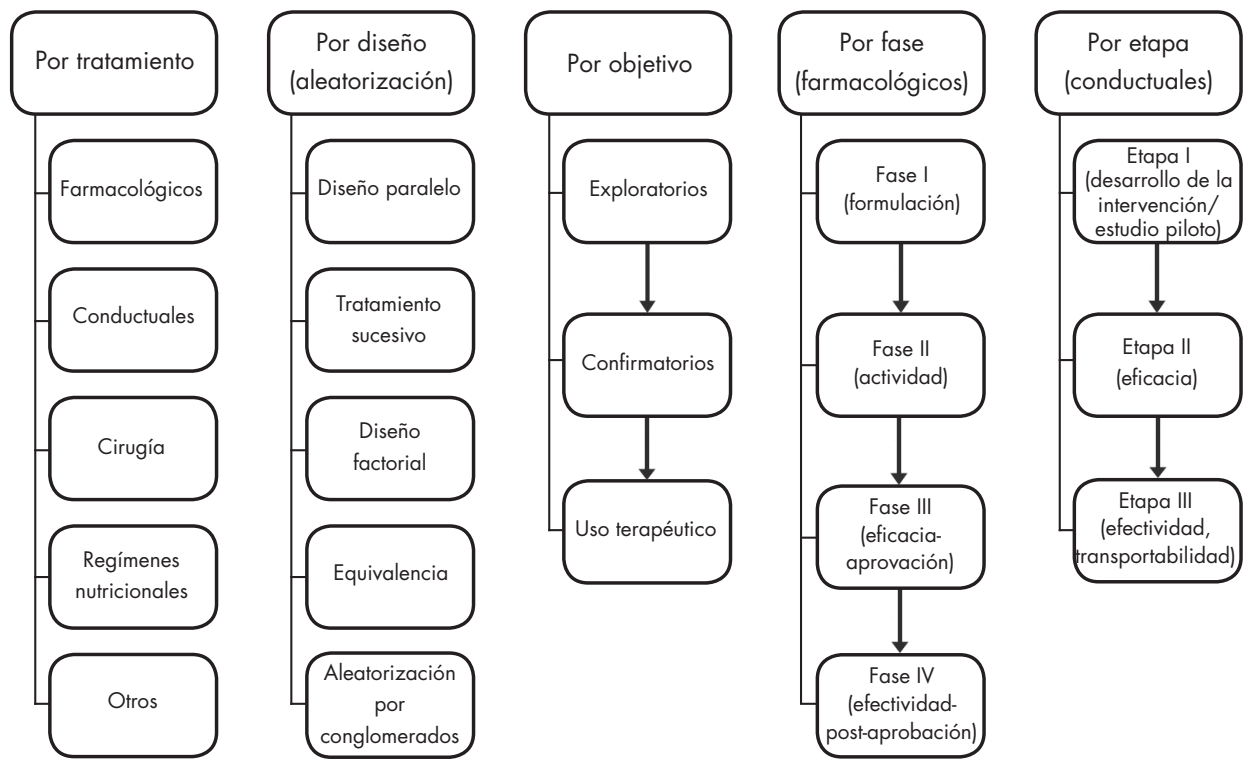

Figura 1. Tipos de ensayos clínicos por criterio de clasificación. 


\section{De acuerdo al tipo de tratamiento}

Pueden clasificarse en ensayos farmacológicos (incluyendo vacunas), terapias conductuales, cirugía, regímenes nutricionales y otros mecanismos terapéuticos. ${ }^{10}$

\section{De acuerdo al diseño}

Esta clasificación incluye, entre otros aspectos, la manera en que los participantes se asignan a las intervenciones. 9,10,19 Cada tipo de ensayo según su diseño se describe con más detalle en el cuadro 1.

\section{De acuerdo al objetivo}

Pueden clasificarse en función de su objetivo o del momento dentro del desarrollo clínico de la intervención en que se encuentran (fase o etapa). En ambas clasificaciones se establece un razonamiento secuencial en el desarrollo de varios ensayos clínicos sobre una misma intervención considerando la influencia de los hallazgos obtenidos previamente. Esta diferenciación secuencial tiene una función descriptiva y no necesariamente implica que un ensayo deba encasillar

Cuadro 1. Clasificación de ensayos por tipo de diseño

\begin{tabular}{ll}
\hline Tipo de Ensayo & \multicolumn{1}{c}{ Descripción } \\
\hline Diseño Paralelo & Es el diseño más común en la práctica. En este \\
& tipo de ensayos los participantes son asignados \\
& a una intervención (ya sea la experimental, o la \\
& intervención control) y son observados de manera \\
& paralela a lo largo de toda la duración del estu- \\
& dio para determinar diferencias en el efecto de las \\
& intervenciones.
\end{tabular}

Diseño de Trata- En este tipo de ensayos los participantes son asigmiento Sucesivo nados de manera aleatoria a distintas secuencias de intervenciones de manera tal que todos reciben todas las intervenciones de estudio, pero en una secuencia distinta.

Diseño Factorial En estos ensayos se asigna de manera aleatoria a todos los participantes a combinaciones distintas de dos o más intervenciones, bajo el entendido de que existe evidencia científica que indica no existe interacción alguna entre éstas y que ambas tienen efectos terapéuticos similares. Bajo este diseño los participantes pueden recibir todas las intervenciones de estudio, sólo una de las intervenciones de estudio, o ninguna (es decir, el tratamiento control o placebo únicamente).

Diseño de Equi- En este tipo de ensayos se comparan dos intervenvalencia ciones de efecto terapéutico similar con la finalidad de demostrar su "equivalencia" terapéutica.

Diseño de Alea- En este tipo se asigna de manera aleatoria a una torización por intervención a grandes grupos de participantes Conglomerados (p.ej. pacientes en un hospital, o de uno o más (Cluster) médicos o terapeutas) en lugar de hacerlo de manera individual por participante.
Cuadro 2. Clasificación de ensayos por objetivos secuenciados

\begin{tabular}{|c|c|}
\hline Tipo de Ensayo & Descripción \\
\hline $\begin{array}{l}\text { 1. Ensayo } \\
\text { Exploratorio }\end{array}$ & $\begin{array}{l}\text { En este tipo de ensayos se estiman cuestiones } \\
\text { como dosis o régimen de tratamiento mínimo para } \\
\text { lograr un efecto terapéutico, se establecen los des- } \\
\text { enlaces clínicos esperados (efecto), y se observan } \\
\text { posibles interacciones entre la intervención nue- } \\
\text { va y otras variables de los participantes. Durante } \\
\text { estos estudios se estima también la seguridad y } \\
\text { tolerancia inicial a la intervención en humanos. }\end{array}$ \\
\hline $\begin{array}{l}\text { 2. Ensayo } \\
\text { Confirmatorio }\end{array}$ & $\begin{array}{l}\text { Estos ensayos se realizan después para demos- } \\
\text { trar o confirmar la eficacia terapéutica de la inter- } \\
\text { vención, establecer una relación dosis-respuesta, } \\
\text { establecer la seguridad de la intervención, y pro- } \\
\text { veer una base adecuada para evaluar la relación } \\
\text { riesgo-beneficio de la intervención para que ésta } \\
\text { pueda incorporarse a la práctica clínica regular. }\end{array}$ \\
\hline $\begin{array}{l}\text { 3. Ensayo de } \\
\text { uso terapéutico }\end{array}$ & $\begin{array}{l}\text { En estos ensayos se terminan de perfeccionar los } \\
\text { análisis de relación riesgo-beneficio, y la dosis o } \\
\text { régimen de tratamiento efectivo y se identifican } \\
\text { los efectos adversos menos comunes. }\end{array}$ \\
\hline
\end{tabular}

su diseño alrededor de ésta pues un mismo ensayo puede presentar un diseño incorporando objetivos de varias fases o etapas de manera simultánea. ${ }^{11,19-22}$ Una descripción más detallada de cada tipo de ensayo según su objetivo se presenta en el cuadro 2.

\section{De acuerdo a la fase de desarrollo en intervenciones farmacológicas y conductuales}

Las intervenciones farmacológicas contemplan cuatro fases secuenciales que van desde una primera prueba experimental (fase I) en humanos, para ver su efecto, hasta pruebas de su interacción con otras drogas o tratamientos disponibles después de que se ha demostrado su uso terapéutico (fase IV). ${ }^{9-11,19,20,23}$ Una descripción más detallada de cada fase se presenta en el cuadro 3. Las intervenciones conductuales, en combinación con los tratamientos farmacológicos, presentan una buena relación costo-efectividad para reducir el consumo, extender el tiempo de abstinencia e impactar en otras áreas de funcionamiento de los pacientes. ${ }^{8,24}$ Onken, Blaine y Battjes (1997) proponen un modelo de investigación en etapas para terapias conductuales análogo al modelo farmacológico y que contempla tres etapas, las cuales se describen a detalle en el cuadro $4 .^{25,26}$

\section{Definición de la población de estudio}

Implícito dentro de la formulación de la pregunta de investigación está el definir la población particular que participará en el ensayo. Para ello se propone plantearse las siguientes preguntas: ¿cuáles son los pacientes que más podrían recibir el beneficio terapéutico de la intervención?, ¿en cuáles es más detectable el efecto de la misma?, ¿quiénes son aquellos en 
Cuadro 3. Clasificación de ensayos por fase de desarrollo. (Ensayos farmacológicos)

\begin{tabular}{ll}
\hline Tipo de & \multicolumn{1}{c}{ Descripción } \\
Ensayo & \\
\hline Fase I & En esta fase se prueba de manera inicial la administración \\
& y efectos de una intervención experimental. Generalmente \\
se realizan en humanos saludables y se observa en la re- & acción o manera en que éstos responden a la intervención \\
(p. farmacodinámica y farmacoquinética de un fármaco) \\
para hacer estimaciones iniciales sobre su dosis tolerable \\
y seguridad (o toxicidad) a corto plazo. También se les \\
conoce como ensayos de "formulación". \\
En esta fase se determina cuál es la dosis mínima con un \\
efecto terapéutico y se estima cuáles son los beneficios \\
obtenidos por la intervención en un grupo pequeño de \\
pacientes con un trastorno específico o cualidades parti- \\
culares a los cuales podría estar dirigida la intervención \\
(p.ej. uso de medicina de mantenimiento en pacientes con- \\
sumidores de opiáceos que inician tratamiento). También \\
se les conoce como ensayos de "actividad". \\
En esta fase se prueba la intervención de manera controla- \\
da en una población más extendida y diversa de pacien- \\
tes (p.ej. con distintos grados de severidad del trastorno, o \\
en distintos estados de desarrollo del mismo) y se compara \\
contra un placebo o la intervención habitual para demos- \\
trar o confirmar su eficacia terapéutica. Durante esta fase \\
es que una nueva intervención demuestra su efecto tera- \\
péutico, se evalúa su seguridad y, finalmente, obtiene su \\
aprobación por los organismos regulatorios aplicables. \\
También se les conoce como ensayos de "eficacia". \\
En esta fase se hacen ensayos para fundamentar u optimi- \\
zar el uso clínico de la intervención dentro de la dosis o \\
régimen previamente autorizado. En estos estudios gene- \\
ralmente se analiza la seguridad de la intervención a largo \\
plazo, su interacción con otras intervenciones (o fármacos) \\
o su efecto en poblaciones de pacientes aún más extensas. \\
También se les conoce como ensayos de "efectividad". \\
\hline
\end{tabular}

los que la aplicación de la intervención representa un alto riesgo de daño o de no-mejora? y (en algunos casos) ise va a incluir a sujetos sanos en el estudio?. La respuestas a estas preguntas permitirán definir criterios de inclusión y criterios de exclusión que deberán seguirse para seleccionar a los pacientes. ${ }^{9,11,19,23}$

\section{ALEATORIZACIÓN DEL TRATAMIENTO}

Uno de los aspectos más importantes en un ECCA es la asignación por azar de tratamientos, ya que con ello se asegura que todos los participantes en el estudio tengan las mismas probabilidades de poder asignarse a la intervención (experimental o control) y sin que los investigadores puedan predecir o influir en este proceso, evitando así un "sesgo de selección". 10,11,19,23,27 Existen distintos métodos de aleatorización acordes al diseño, al tamaño de la muestra y a los objetivos de cada ensayo, los cuales se describen en el cuadro 5. .-11,19,23,27 $^{-1}$
Cuadro 4. Clasificación de ensayos por etapa de desarrollo en tratamientos conductuales

Tipo de

Estudio Descripción

Etapa I Es la etapa inicial en la cual se busca la innovación clínica mediante el estudio de los cambios en el comportamiento que se espera de los pacientes en la terapia y la definición de los componentes y cualidades únicos de la intervención que tengan utilidad clínica. Durante esta etapa se prueba el efecto terapéutico de una intervención y se genera el Manual de la Intervención, un plan de entrenamiento en la misma, y una medida de adherencia y competencia en su aplicación que puedan utilizarse en las siguientes etapas. También se les conoce como "Estudios de innovación clínica".

Etapa II En esta etapa se llevan a cabo ensayos clínicos en una muestra más extensa de pacientes "ideales" para la intervención y en condiciones altamente controladas (con el fin de asegurar que el efecto terapéutico se debe precisamente a la intervención de estudio). Su propósito es probar la eficacia de una intervención ya desarrollada contra otros tratamientos o un placebo. También se les conoce como "Estudios de Eficacia".

Etapa III Esta etapa se dedica a la realización de ensayos clínicos enfocados en estudiar la efectividad de una intervención cuya eficacia se ha demostrado en estudios previos, una vez que se aplica en los escenarios comunitarios de atención. La investigación en esta etapa busca responder preguntas acerca de la interacción entre los componentes de una intervención y variables presentes en los escenarios comunitarios de atención (p.ej. necesidades de entrenamiento de los terapeutas). Las preguntas de investigación en esta fase son en torno a la implementación, aceptabilidad (o adopción), generalizabilidad, transportabilidad y/o relación costo-beneficio de la intervención en función de analizar la pertinencia, viabilidad o sustentabilidad de su incorporación a la práctica clínica regular. También se les conoce como "Estudios de Efectividad".

\section{Análisis estadístico e interpretación de resultados}

Su aplicación permite minimizar las fuentes de error en la interpretación de los resultados, por lo tanto es imprescindible sistematizar los procedimientos para lograr el análisis de resultados válidos y confiables. Dentro de las consideraciones estadísticas que se deben tomar en cuenta en el desarrollo de un ensayo clínico aleatorizado, existen tres temas fundamentales a desarrollar: 1) la planeación del tamaño de la muestra, 2) los procedimientos de aleatorización (explicados en la sección anterior) y 3) el plan de análisis estadístico $^{27}$ (figura 2). De esta forma, una parte fundamental de los ECCA es implementar una estrategia en función de los objetivos del estudio, ${ }^{28,29}$ y fundamentada en el planteamiento del diseño. ${ }^{30}$ Adicionalmente, dentro del protocolo de investigación puede describirse brevemente el software utilizado para el análisis de datos, nombrar la utilización de técnicas estadísticas preliminares para asegurar el cumplimiento de los supuestos estadísticos o, en el caso de que no 
Cuadro 5. Métodos más comunes de aleatorización

\begin{tabular}{ll}
\hline $\begin{array}{l}\text { Método de } \\
\text { Aleatorización }\end{array}$ & \multicolumn{1}{c}{ Descripción } \\
\hline Aleatorización & Es la forma más sencilla de asignación aleatoria y \\
Simple & equivalente a cualquier sorteo o al lanzamiento de \\
& una moneda. En la práctica, este método raramente \\
& se utiliza ya que puede generar un desbalance en el \\
& número de pacientes asignado a cada grupo cuando \\
& la muestra es pequeña, o es muy alto el riesgo de que \\
& genere algún sesgo accidental en la asignación de \\
& intervenciones.
\end{tabular}

Aleatorización Este método se utiliza con la finalidad de asegurar un en bloques ba- balance en el número de pacientes asignado a cada lanceados intervención del estudio.

Consiste en los siguientes pasos:

a. Generación bloques con todas las combinaciones posibles entre las intervenciones de estudio a los cuales se les asigna un número.

b. Cada bloque será una "secuencia" en la cual se asignará la intervención a un número determinado de participantes.

c. Por medio de un sorteo, se asignará un bloque a distintos grupos de participantes hasta completar la muestra.

Este método es muy utilizado en la práctica. Una posible desventaja es que, si la asignación de intervenciones no es ciega, puede que dentro de un grupo se pierda la cualidad "impredecible" de la asignación individual de tratamientos.

Aleatorización Este método se utiliza si los investigadores cuentan estratificada con evidencia científica acerca de alguna variable en los pacientes que tengan un valor predictivo sobre su respuesta a la intervención (p.ej. edad, género, severidad de la adicción, tiempo de abstinencia, comorbilidad psiquiátrica) con la finalidad de asegurar una muestra balanceada de pacientes con esa variable en todos los grupos.

Generalmente se lleva a cabo determinando un número de "estratos" acorde a los valores posibles de cada variable que se identificó tiene un poder predictivo (o un potencial como confesor), generando una lista de combinaciones entre los estratos de todas las variables que constituirán "sub-estratos". Después se realiza una asignación aleatoria simple o de bloques balanceados de la intervención para cada grupo de pacientes que se incluya en cada "sub-estrato".

Para llevar a cabo este método de aleatorización, es preciso contar con una muestra de participantes lo suficientemente extensa que la haga viable para obtener un número igual de pacientes con cada combinación de variables.

Aleatorización Es una variación de cualquiera de los métodos de dinámica aleatorización mencionados antes, salvo que en estos casos la probabilidad con que un participante sea asignado a un grupo de intervención u otro se va "balanceando" o "ajustando" en función de ciertas variables (p.ej. género, edad) conforme se van conformando los grupos de intervención con la finalidad de asegurar que éstos son comparables entre sí.

Es importante recalcar que a pesar de este balanceo, los investigadores que empleen este método jamás deberán ser capaces de predecir a qué intervención será asignado un paciente a partir de sus características y que este método tampoco asegura que los grupos sean perfectamente balanceados.
Ejemplo

En un ensayo donde se comparan los efectos de una Intervención $A$ con los de una Intervención $B$, se asigna a cada participante un número entre el 1 y el 10 generado al azar en una computadora.

- Si el participante obtiene un número entre el 1 y el 5 se le asigna el Tratamiento A.

- Si obtiene un número entre el 6 y el 10 se le asigna el Tratamiento B. Todos los participantes tienen un $50 \%$ de probabilidad de ser asignados a un tratamiento o a otro.

En un ensayo donde se comparan los efectos de una Intervención A con los de una Intervención B:

a. Se generan los siguientes 6 bloques de combinaciones entre las letras $A$ y $B$ :
1. $A A B B$
2. $B B A A$
4. $B A B A$
3. $A B A B$
5. $A B B A$
6. $B A A B$

b. Se generan grupos de 4 participantes.

c. A cada grupo de participantes se le asigna por azar un número del 1 al 6. Si el primer grupo de participantes (Ptpes. 1-5) obtiene un 3 , se les asignan las intervenciones en la secuencia correspondiente al bloque $5(A B A B)$ :

- Ptpe. 1: Intervención $A$

- Ptpe. 2: Intervención B

- Ptpe. 3: Intervención A

- Ptpe. 4: Intervención B

d. Se continúa asignando números del 1 al 6 al azar a los siguientes grupos hasta completar la muestra.

En un estudio se comparan los efectos de una Intervención $A$ con los de una Intervención B para disminuir la frecuencia de consumo en el paciente. Hay evidencia de que la respuesta al tratamiento puede verse afectada por las siguientes características del paciente:

- Presenta una comorbilidad psiquiátrica al comenzar tratamiento.

- El alcohol es su principal sustancia de consumo.

I. Se generan los siguientes dos "estratos":

1.a. Con comorbilidad psiquiátrica.

1.b. Sin comorbilidad psiquiátrica.

2.a. Consumo principal de alcohol

2.b. Sin consumo principal de alcohol.

II. Se agrupa a los participantes en los siguientes 4 "sub-estratos".

1.a. Pacientes con comorbilidad psiquiátrica + 2.a. Consumo principal de alcohol.

1.b. Pacientes sin comorbilidad psiquiátrica +2 .a. Consumo principal de alcohol.

1.a. Pacientes con comorbilidad psiquiátrica $+2 . b$. Sin consumo principal de alcohol.

1.b. Pacientes sin comorbilidad psiquiátrica $+2 . b$. Sin consumo principal de alcohol.

III. Se conduce una aleatorización simple de manera independiente en cada "sub-estrato".

En un ensayo donde se comparan los efectos de una Intervención A con los de una Intervención B se ha decidido conducir una aleatorización en la cual las probabilidades de que un participante sea asignado a un grupo o a otro se irán modificando de acuerdo a las siguientes variables:

- Sexo (Masculino; Femenino)

- Edad (Entre 18 y 36; mayores de 37).

- Principal sustancia de consumo (alcohol, cocaína, marihuana, otros). De manera que si el primer paciente aleatorizado fue una mujer, mayor de 36 y consumidora de marihuana y fue asignada por aleatorización simple a la Intervención $A$; al siguiente paciente con estas mismas características se le alterarán sus probabilidades de aleatorización para que sea más probable que se le asigne la Intervención B. 


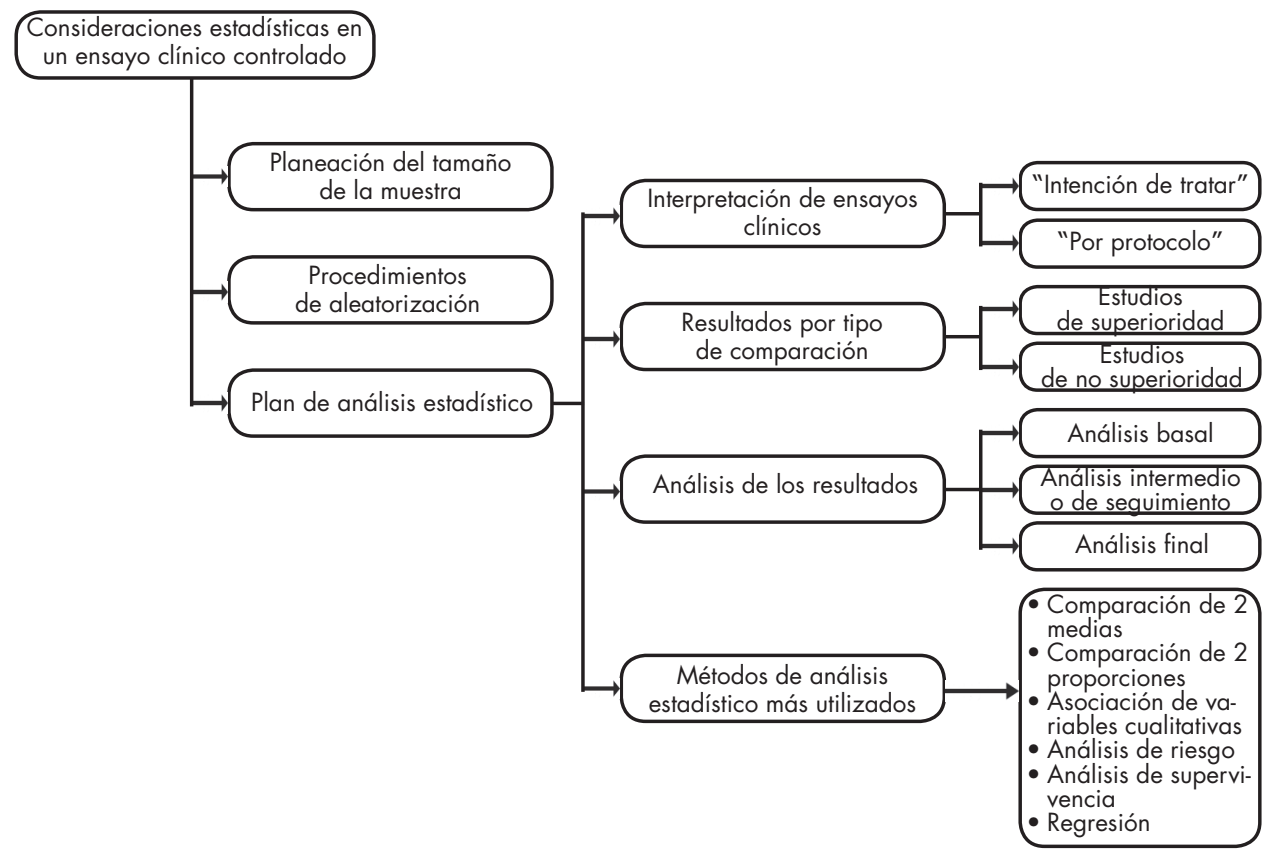

Figura 2. Consideraciones estadísticas en un ensayo clínico controlado.

se cumplan, buscar pruebas equivalentes que no presenten dichas limitaciones. ${ }^{31}$

Para la interpretación de los resultados existen al menos dos métodos: el primero se refiere al principio de "intención de tratar", el cual considera la inclusión de todos los participantes aleatorizados en el análisis, a fin de mantener la comparabilidad entre los grupos de intervención; $; 0,23,30,32$ el segundo es el "análisis por protocolo", en el que únicamente se considera en los resultados a los que cumplieron con el protocolo a lo largo del estudio ${ }^{10,30}$ (cuadro 6). Por otro lado, los ECCA pueden tener dos tipos de comparación de resultados: los estudios de superioridad y los de no inferioridad. Los primeros identifican superioridad de una intervención en términos de eficacia; los segundos muestran que el tratamiento experimental no es peor que el tratamiento estándar. Ambos tipos de estudio se explican con mayor detalle en el cuadro 7. La opción a elegir dependerá de la relevancia clínica y no de la estadística. ${ }^{10,30}$

En un ensayo clínico controlado aleatorizado se lleva a cabo más de un análisis de datos a lo largo del estudio, en general éstos pueden dividirse en tres: análisis basal, intermedio o de seguimiento y análisis final. Cabe señalar que cada uno constituye un elemento imprescindible para la obtención válida y confiable de los resultados finales ${ }^{31}$ (cuadro 8).

\section{Monitoreo clínico o de tratamiento}

Una medida esencial para asegurar la validez interna en los ECCA es poder comprobar que la intervención terapéutica es administrada a todos los participantes en cumplimiento o adherencia con su diseño y evitar el "sesgo de intervención"
Cuadro 6. Interpretación de ensayos clínicos

\begin{tabular}{cc}
\hline Método & Principio de "Intención de Tratar" \\
\hline Descripción El análisis de los datos es realizado mediante la inclu- \\
sión de todos los participantes aleatorizados en el estu- \\
dio, por lo que los eventos que surgen a lo largo de éste \\
(falta de apego al tratamiento, abandono, muerte, entre \\
otros) son contados contra el tratamiento asignado (Pe- \\
duzzi P. y cols., 2002; Green S., 2002; Lazcano-Ponce \\
E. y cols., 2004; Lazcano-Ponce E. y cols., 2009).
\end{tabular}

Ventajas Mantiene la comparabilidad entre los grupos de intervención.

Minimiza el sesgo en los resultados del estudio, ya que el tamaño de la muestra, definido previamente, no se ve alterado.

Al considerar a todos los participantes aleatorizados evita favorecer a cualquiera de los grupos de intervención. Provee una estimación conservadora del efecto de tratamiento.

Desventajas No permite obtener una evaluación verdadera de la eficacia del tratamiento, aunque sí de la efectividad del mismo.

\section{"Análisis por Protocolo"}

Descripción El análisis de los resultados considera, únicamente, al subgrupo de participantes que cumplieron con el protocolo a lo largo del estudio. Este método de análisis debe diseñarse a priori, identificando los criterios para considerar el cumplimiento suficiente del protocolo (Lazcano-Ponce E. y cols., 2004; Lazcano-Ponce E. y cols., 2009).

Ventajas Este método facilita que un tratamiento muestre eficacia adicional.

Desventajas En ocasiones el apego al protocolo durante el estudio puede estar directamente relacionado con la intervención asignada, lo cual puede introducir un sesgo importante en los resultados. 
Cuadro 7. Resultados por tipo de comparación

\begin{tabular}{cl}
\hline Tipo de Comparación & \multicolumn{1}{c}{ Descripción } \\
\hline Estudios de Superioridad & $\begin{array}{l}\text { Este tipo de estudios de comparación } \\
\text { tienen por objetivo identificar la superio- } \\
\text { ridad, en términos de eficacia, de una } \\
\text { intervención frente al placebo o trata- } \\
\text { miento control, ya sea porque produce } \\
\text { claramente mejores resultados o porque } \\
\text { muestra un efecto de dosis-respuesta. } \\
\text { Estos estudios pretenden mostrar que el } \\
\text { tratamiento experimental no es peor que } \\
\text { el tratamiento estándar, sin embargo, } \\
\text { para llevar a cabo este tipo de compa- } \\
\text { ración es indispensable que el estudio } \\
\text { sea conducido con la más alta calidad, } \\
\text { a fin de permitir la identificación de dife- } \\
\text { rencias significativas entre los grupos. }\end{array}$ \\
\hline
\end{tabular}

tanto en los ensayos farmacológicos como en los conductuales. ${ }^{9,11,33}$ En los ensayos farmacológicos se debe asegurar el cumplimiento o adherencia, lo que implica contar con un procedimiento para monitorear que a todos los participantes se les administra el fármaco correcto y que reciben dicho fármaco siguiendo las indicaciones prescritas con opciones tales como muestras de sangre, marcadores biológicos asociados, monitoreo de los suministros, contabilización de píldoras, auto-reportes o revisión de registros. ${ }^{9,11,19,33} \mathrm{La}$ elección de la estrategia deberá basarse en un análisis de su confiabilidad y usabilidad con respecto al diseño y objetivos del estudio así como las características particulares de la población de pacientes y la intervención del estudio. ${ }^{34}$

En las intervenciones conductuales la validez interna se afecta cuando existe poca o nula diferenciación entre los

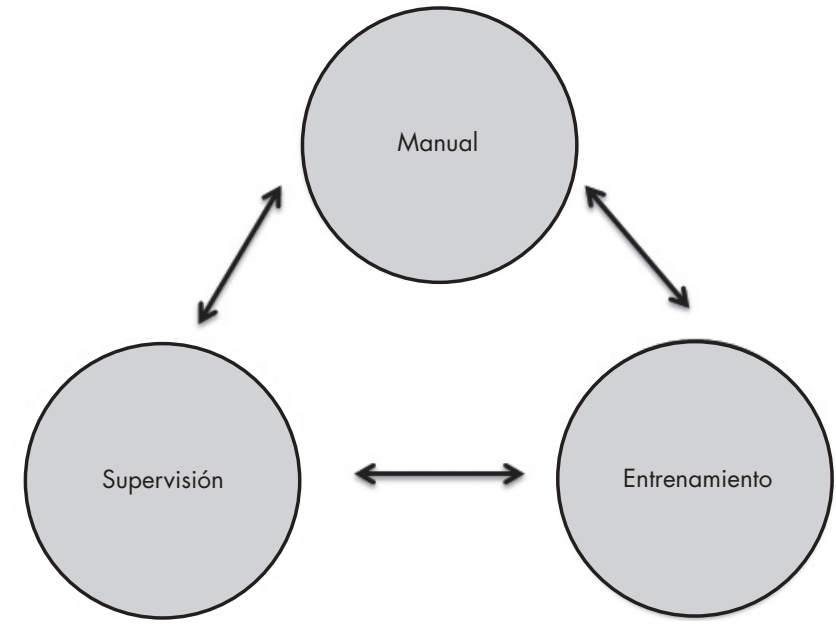

Figura 3. Elementos a considerar dentro del aseguramiento de la integridad de terapias conductuales en ensayos clínicos aleatorizados.

tratamientos, por intervenciones difusas y por variaciones en la forma en que se lleva a cabo la intervención. ${ }^{35}$ Por ello, la estandarización de la maniobra constituye un elemento crucial. ${ }^{36-38}$ Hay al menos tres elementos para asegurar la integridad de las terapias en los ECCA: el manual de intervención, el entrenamiento y la supervisión ${ }^{26,37,39,40}$ (figura 3). La creación de un manual para una nueva terapia conductual ocurre en la etapa I del modelo de etapas de las terapias conductuales mencionado previamente (cuadro 8), ${ }^{25}$ e implica la definición de los elementos críticos del nuevo tratamiento. ${ }^{26,41}$ Por lo que respecta al entrenamiento, existen diferentes retos a considerar. Éstos incluyen el grado de motivación, interés, experiencia y educación previa de los terapeutas, la

Cuadro 8. Análisis de resultados

Análisis Basal

Descripción Es el primer análisis de las variables que se lleva a cabo después de la aleatorización de los participantes a los diferentes grupos de tratamiento.

Utilidad En ocasiones se presentan diferencias importantes en algunas variables principales, que de no considerarse, podrían atribuirse erróneamente al efecto de la intervención; por lo tanto, la identificación de dichas variables, por medio del análisis basal, permite realizar comparaciones, controlando dicho efecto mediante pruebas de análisis estadístico, entre ellas, la covarianza.

Análisis Intermedio

Descripción Es el análisis de las variables que se realiza a lo largo del estudio, también es llamado análisis de seguimiento y pueden llevarse a cabo uno o más análisis intermedios, dependiendo del tiempo de duración del estudio.

Utilidad El análisis intermedio permite observar la evolución de las variables a lo largo del tiempo, con lo cual es posible establecer una relevancia clínica y estadística comparando los grupos de tratamiento experimental y control.

Además, en el marco ético de la investigación, el análisis intermedio permite identificar resultados preliminares que pueden impactar de manera importante en los participantes, de esta forma, en caso de que el tratamiento experimental resulte perjudicial debido a efectos adversos inesperados, es vital la interrupción del estudio o, en sentido inverso, en caso de que la intervención sea evidentemente mejor que el tratamiento control, se debe suspender el estudio para poner a disposición de todos los pacientes afectados por determinada enfermedad o padecimiento la nueva intervención.

$$
\text { Análisis Final }
$$

Descripción Tiene lugar una vez que termina el tiempo del ensayo y se procede a realizar un análisis definitivo de los resultados.

Utilidad Al estar contenido el plan de análisis final en el protocolo de investigación, los contrastes están previstos y la probabilidad de que las diferencias encontradas se deban al azar es menor. 


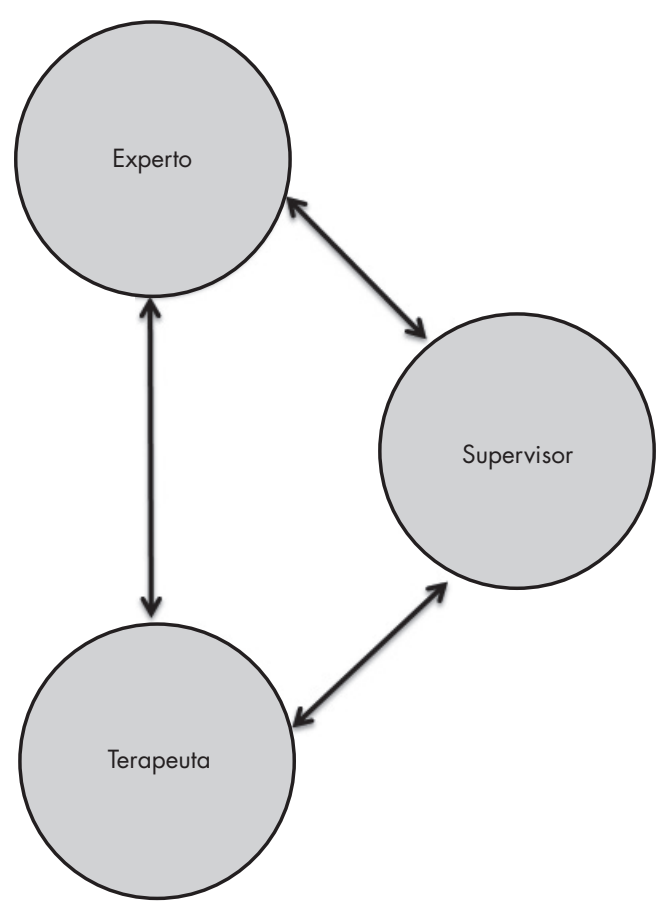

Figura 4. Perspectiva de entrenamiento y supervisión train the trainer.

aproximación terapéutica con que usualmente llevan a cabo su práctica y el formato y duración de la capacitación ofrecida. ${ }^{42}$ Beidas y Kendall (2010) proponen que un entrenamiento didáctico sin monitoreo ni evaluación de la práctica subsecuente de las habilidades aprendidas tiene poco o ningún efecto en la destreza y/o adherencia de los terapeutas a la maniobra aprendida. En este sentido, el entrenamiento y la supervisión se convierten en dos procesos muy ligados entre sí, ya que bajo esta óptica se aprende por medio de talleres prácticos y con supervisión continua y retroalimentación sobre el trabajo realizado en las sesiones. ${ }^{40,43-45}$ Dentro de este estilo se distinguen dos diferentes aproximaciones: expert-led y train-the-trainer. En la perspectiva train-the-trainer (figura 4), o entrenar al entrenador, un experto entrena a otros terapeutas en la maniobra y les enseña cómo entrenar a su vez a otras personas en su centro de tratamiento, sosteniendo sesiones de retroalimentación, monitoreo y supervisión constantes. ${ }^{46}$ En esta aproximación, a diferencia del expert-led (figura 5), se tiene la ventaja de contar con un profesional que conoce el tratamiento y que al mismo tiempo cuenta con la capacidad de entrenar y supervisar a otros profesionales. Es así que se convierte en un supervisor cuya práctica favorece la fidelidad a la maniobra terapéutica, y por ende protege la validez interna del estudio. ${ }^{47}$

El entrenamiento, la acreditación y la supervisión continua están ligados al proceso de transferencia de entrenamiento y a la transferencia de tecnología, traduciéndose esto en la diseminación y utilización de maniobras eficaces $y$ efectivas en escenarios reales, ${ }^{47,48}$ lo que significa un gran reto en la atención del consumo de sustancias. ${ }^{42}$

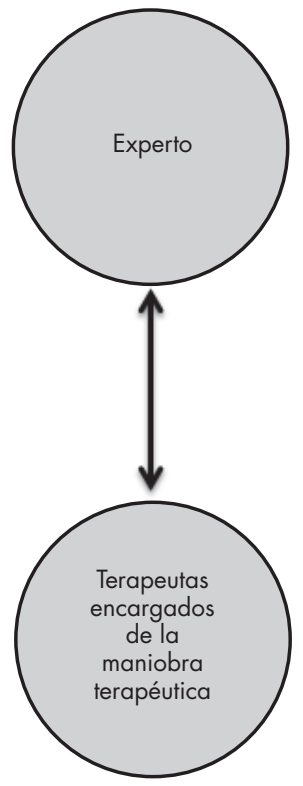

Figura 5. Perspectiva de entrenamiento expert-led.

\section{Monitoreo de seguridad}

La evidencia que respalda la decisión clínica de utilizar una intervención sobre otra se constituye en la misma dimensión tanto por su eficacia o efectividad demostrada como por sus posibles efectos iatrogénicos, o no-deseados, en todas las poblaciones de pacientes donde potencialmente pueda aplicarse. Por esto es un requisito regulatorio en la conducción de ensayos clínicos el contar con un mecanismo o sistema de recolección de datos acerca de todos estos efectos en los participantes. Durante la conducción de un ECCA a todos estos efectos no-deseados se les identifica bajo la designación de "eventos adversos". 9,10,49 En el tratamiento de las adicciones y de otros trastornos de la salud mental los pacientes tienen un alto riesgo de presentar afecciones renales, cardiacas o respiratorias e infecciones asociadas al consumo; así como accidentes, conductas sexuales de riesgo, comportamiento violento o problemas con la ley debidos al abuso de sustancias; además de una exacerbación de la sintomatología psiquiátrica, o incluso conductas suicidas, causadas por el consumo de alcohol o de drogas ${ }^{1,5,8,15,50,51}$ o dificultades en el funcionamiento familiar, laboral o social del sujeto. ${ }^{52}$ La conducción de un ECCA debe contemplar un plan acorde para la identificación, evaluación, seguimiento y reporte de posibles eventos adversos. Los mecanismos o herramientas que se utilicen para llevar a cabo este monitoreo de seguridad deberán ser parte del protocolo de investigación. 9,52 En el cuadro 9 se muestra una propuesta de las distintas categorías de eventos adversos que deben identificarse en los participantes de un ECCA. ${ }^{9}$ Todo conocimiento que tengan los investigadores acerca de los posibles riesgos o eventos adversos que se detecten y que están relacionados con la intervención 
Cuadro 9. Categorías de eventos adversos

\begin{tabular}{|c|c|}
\hline Categoría & Descripción \\
\hline $\begin{array}{l}\text { Eventos Adversos } \\
\text { Serios }\end{array}$ & $\begin{array}{l}\text { Son todos aquellos eventos adversos que resultan en } \\
\text { fallecimiento del paciente, constituyen una amenaza } \\
\text { a su vida, requieren su hospitalización (o la prolon- } \\
\text { gación no planeada de la hospitalización existente), } \\
\text { da como resultado una incapacidad persistente, irre- } \\
\text { versible o significativa del paciente, es una anoma- } \\
\text { lía congénita o defecto de nacimiento, o requieren } \\
\text { una intervención médica o psiquiátrica para preve- } \\
\text { nir cualquiera de las situaciones anteriores. }\end{array}$ \\
\hline
\end{tabular}

Eventos Adversos Son todos aquellos signos, síntomas o empeoraRegulares miento de condiciones preexistentes que se detecte en el participante ya sea mediante su autoreporte, o por observación de resultados clínicos u observación del entrevistador o clínico tratante. Estos eventos pueden variar en su severidad o impacto en la salud del participante.

Eventos Adversos Son todos aquellos eventos adversos (serios, generade Interés Especial les o de cualquier severidad) que para fines del protocolo serán identificados y clasificados de manera especial debido a que revisiones de la evidencia científica, o por determinación de un consejo experto consultado para estos fines, han demostrado que pueden tener un impacto sobre la interpretación de los resultados o la aplicabilidad de la intervención de estudio. En otras palabras, pueden estar relacionados o asociados con la intervención de estudio.

de estudio debe ser informado a las instancias regulatorias aplicables (p.ej. comités de ética institucionales) y, si se considera necesario, a todos los participantes del estudio.

\section{ADMINISTRACIÓN DE DATOS}

El propósito del ECCA es generar datos a partir de los cuales sea posible proveer una respuesta a una pregunta de investigación. Uno de los procesos asociados a esta tarea es la administración de datos, que es un proceso que se inicia desde antes del reclutamiento. Su finalidad es asegurar la calidad de los datos generados lo cual, en buena medida, depende de la claridad con la que se han definido los objetivos y el análisis estadístico del estudio. ${ }^{9}$ Los resultados que se reporten al finalizar son tan buenos como lo sea la calidad de los datos recaudados y analizados. Se define como "buen" resultado a aquél que suministra las respuestas correctas a las preguntas inicialmente planteadas, no a los resultados positivos o estadísticamente significativos. ${ }^{53} \mathrm{El}$ plan de administración de datos abarca desde el diseño de los Formularios de Reporte de Caso (FRC) hasta la entrega de datos para el análisis estadístico. Por ello los datos necesitan ser constantemente monitoreados durante el estudio y se debe recolectar el máximo de datos posibles para su análisis. ${ }^{54}$ La introducción de nuevas tecnologías en el proceso de administración de datos ha reducido significativamente los errores que se producían al recolectarlos mediante los FRC al sustituirlos por los FRC electrónicos. Actualmente se utilizan los denominados Sistemas de Administración de Datos Clínicos (CDMS - Clinical Data Management Systems). Del mismo modo, la inclusión de la Internet permite que los CDMS estén disponibles en línea, permitiendo la recolección y/o monitoreo de datos en distintos sitios y horarios.

\section{CONSIDERACIONES ÉTICAS}

\section{"Equipoise" o principio de incertidumbre sobre los tratamientos}

La primera implicación ética asociada a los ensayos clínicos tiene que ver con la asignación aleatoria de intervenciones terapéuticas. Si los investigadores o clínicos que colaboran en el reclutamiento y tratamiento de pacientes para un ensayo clínico tienen "certidumbre" acerca de las ventajas terapéuticas de una intervención sobre otra, tienen la obligación ética de proveer al paciente aquella intervención que tenga más posibilidades de representar un beneficio para él. ${ }^{49,55}$ Freedman (1987) ha propuesto el principio ético de "Equipoise" el cual establece que para que un ECCA se realice, las intervenciones de estudio deberán ser "equiparables" entre sí en cuestiones del beneficio terapéutico que representan para el paciente. Esto implica que los investigadores, al no tener control sobre qué intervención terapéutica se proveerá a los participantes, deben estar en un estado de "genuina incertidumbre" acerca del posible beneficio terapéutico que recibirá el participante del estudio, independientemente de la intervención que se le asigne. La función última de la investigación sobre la intervención sería resolver esa incertidumbre. ${ }^{55-57}$

\section{Plan de calidad y protección de los participantes}

Aunque la investigación de tratamientos data desde antes, ${ }^{59}$ no fue sino hasta el siglo XX que se reconoció la necesidad de contar con estándares y regulaciones en función de asegurar la protección de los humanos participantes en una investigación. 9,10,49 En el cuadro 10 se muestra a detalle una relación de las principales guías desarrolladas para estos fines. ${ }^{58-64}$ Toda investigación sobre una intervención lleva implícita un importante dilema ético en la calidad de sus resultados, pues éstos fundamentarán una decisión sobre la salud, tanto de los participantes como de los pacientes que potencialmente se verán beneficiados por ésta. En un esfuerzo de estandarización conducido por varias agencias reguladoras de medicamentos en varios países (englobadas como la Conferencia Internacional de Armonización o ICH, por sus siglas en inglés) sobre la investigación con sujetos humanos, se establecieron los Lineamientos de las Buenas Prácticas Clínicas (BPC), en 1990. ${ }^{58}$ 
Cuadro 10. Evolución de la normatividad aplicable

\begin{tabular}{|c|c|}
\hline Normatividad aplicable & Descripción \\
\hline Código de Núremberg (1947) & $\begin{array}{l}\text { Plantea y delimita la (i)legitimidad de la investigación médica; así como el proceso de consentimiento } \\
\text { informado y la ausencia de coerción en la participación de los sujetos. }\end{array}$ \\
\hline Declaración de Ginebra (1948) & Se trata de un documento en el que el médico profiere una práctica profesional ética y honorable. \\
\hline Declaración de Helsinki (1964) & $\begin{array}{l}\text { La asamblea médica mundial publicó dicha declaración presentándola como "una propuesta de principios } \\
\text { éticos para investigación médica en seres humanos, incluida la investigación del material humano y de } \\
\text { información identificables." (Intro, punto 1: http://www.wma.net/es/30publications/10policies/b3/). }\end{array}$ \\
\hline $\begin{array}{l}\text { Ley Nacional de Investigación (E.U.) } \\
\text { (1974) }\end{array}$ & $\begin{array}{l}\text { Reporte Belmont 1979; dictamina tres principios éticos fundamentales en la investigación con seres } \\
\text { humanos: respeto, beneficencia y justicia. }\end{array}$ \\
\hline Ley General de Salud México (1983) & Reglamento de la ley general de salud en materia de investigación para la salud (1987). \\
\hline $\begin{array}{l}\text { Conferencia Internacional sobre Armo- } \\
\text { nización (ICH; acuerdo entre E.U., Ja- } \\
\text { pón y la Unión Europea) (1990) }\end{array}$ & $\begin{array}{l}\text { Se trata de la reunión de las autoridades reguladoras y la industria farmacéutica de Europa, Japón y } \\
\text { E.U. para discutir los aspectos científicos y técnicos del registro de medicamentos. }\end{array}$ \\
\hline $\begin{array}{l}\text { Buenas Prácticas Clínicas (BPC)-Docu- } \\
\text { mento de las Américas (2005) }\end{array}$ & $\begin{array}{l}\text { Según } \mathrm{ICH} \text { : "norma internacional de calidad científica y ética dirigida al diseño, realización, regis- } \\
\text { tro y redacción de informes de ensayos que implican la participación de seres humanos" (CPMP/ } \\
\mathrm{ICH} / 135 / 95) \text {. }\end{array}$ \\
\hline
\end{tabular}

Todo ensayo clínico debe contemplar el desarrollo de un plan de monitoreo de calidad en donde se registre un seguimiento de la evolución del sujeto participante en el estudio. El objetivo de dicho plan, según las BPC, es asegurar que: a) estén protegidos los derechos y el bienestar de los sujetos humanos, b) los datos reportados del estudio estén completos, sean precisos y verificables, c) la conducción del estudio esté en conformidad con el diseño y procedimientos reportados por los investigadores, con las BPC, y con las regulaciones aplicables ${ }^{63,64}$ (figura 6). Un punto esencial es la constante auditoría del ensayo desde su diseño, planeación, ejecución y análisis y reporte de resultados en la que deben estar involucrados autoridades, patrocinadores, investigadores y colaboradores relacionados con el estudio. En la figura 7 se enumeran todos los componentes que deben estar involucrados en la investigación para el aseguramiento de la calidad.

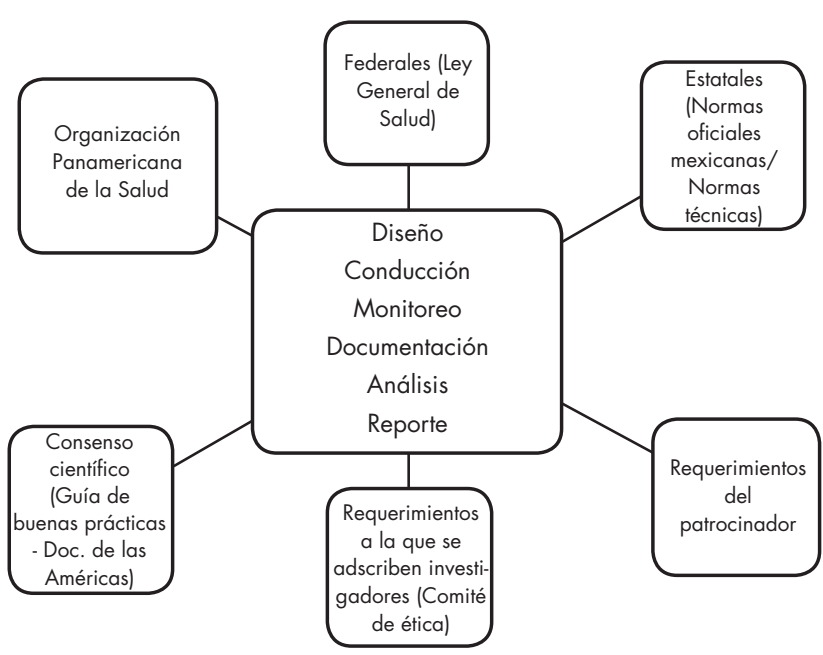

Figura 6. Regulaciones aplicables a los procesos de un ensayo clínico.

\section{Consentimiento informado y confidencialidad}

En México, la Ley General de Salud, en su artículo 20 del reglamento en materia de investigación para la salud, define el consentimiento informado como: "el acuerdo por escrito, mediante el cual el sujeto de investigación o, en su caso, su representante legal autoriza su participación en la investigación, con pleno conocimiento de la naturaleza de los procedimientos y riesgos a los que se someterá, con la capacidad de libre elección $y$ sin coacción alguna". ${ }^{62}$ En el consentimiento informado se encuadran las particularidades de la participación del sujeto en la investigación, y se documenta la decisión del paciente de participar en ella. ${ }^{65}$ Es responsabilidad de los investigadores redactarlo de manera clara y sin ambigüedades, siguiendo una estructura de contenidos. ${ }^{63,64}$ En el cuadro 11 se muestra una propuesta de contenido para el documento

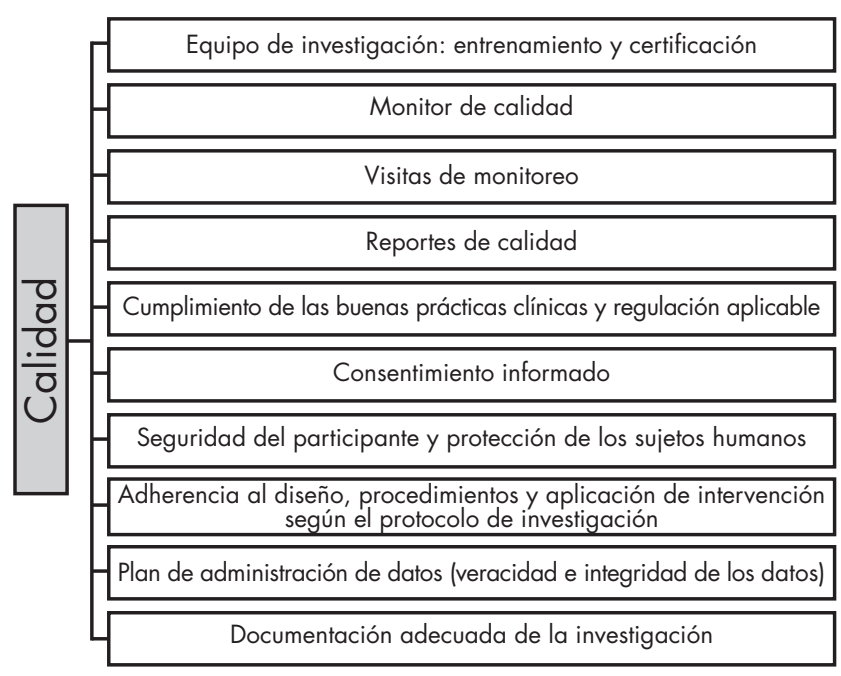

Figura 7. Componentes para asegurar la calidad de la investigación. 
Cuadro 11. Propuesta de estructura de contenidos para el documento de consentimiento informado (CI)

I. Título del estudio

II. Propósito u objetivos del estudio

III. Procedimientos de la investigación

IV. Posibles riesgos e incomodidades

V. Posibles beneficios, alternativas

VI. Posibles costos de su participación

VII. Incentivos a recibir por su participación (si aplica), garantías sobre la confidencialidad de su participación

VIII. Derechos de retirarse

IX. Fecha de expiración para el mantenimiento de registros, información de contacto de los investigadores

X. Sección para registrar la firma de:

i. participante o su representante legal

ii. la persona que obtiene el $\mathrm{Cl}$

iii. dos testigos

de consentimiento informado a partir de lo establecido en la Ley General de Salud y las Buenas Prácticas Clínicas. ${ }^{62,63}$ Se debe entregar al participante un duplicado del documento de consentimiento informado firmado, así como hacer público el procedimiento mediante el cual se obtuvo. .1,49,62 $^{\text {, }}$

Una investigación clínica trata usualmente de cuestiones de naturaleza personal de los participantes cuyo manejo es delicado. En el caso particular de las investigaciones sobre trastornos por consumo de sustancias esta información adquiere un grado mayor de delicadeza, pues los participantes se exponen a sufrir consecuencias legales o sociales si se llegase a revelar su identidad como participantes en un ensayo clínico. ${ }^{49}$ Toda información que pueda identificar a los participantes debe mantenerse bajo estricta confidencialidad. Es indispensable que los investigadores informen a los participantes antes de su reclutamiento, así como a las instancias regulatorias aplicables, acerca de los procedimientos que se seguirán para garantizar la confidencialidad de los participantes así como sobre los casos en que ésta puede estar limitada (p.ej. si durante su participación se identifica un riesgo suicida en el paciente se deberá notificar a sus familiares y responsables clínicos para que se le derive a una atención especializada). ${ }^{10,11,49}$

\section{REDACCIÓN DEL PROTOCOLO}

El protocolo de investigación es un documento en el cual los investigadores hacen pública todas las consideraciones tomadas alrededor de la conducción de un ECCA para su revisión por las instancias regulatorias, patrocinadores y comunidad científica en general. Dicho documento debe describir de manera detallada todos los elementos y procedimientos que se van a realizar. Aunque no existe un consenso universal sobre lo que debe contener un documento de protocolo, pues puede variar de acuerdo a cada ensayo, se han hecho esfuerzos por brindar un estándar de los contenidos mínimos que se deben incluir, entre los que se encuentran: los antecedentes y razonamiento del estudio, objetivos y pregunta de investigación, población a estudiar, intervenciones de estudio, método de reclutamiento y aleatorización de participantes, plan de análisis estadístico y las consideraciones éticas; adicionalmente pueden incluirse el plan de diseminación de la intervención una vez que se haya demostrado su efecto y el plan de administración del estudio ${ }^{9-11,27,66}$ (Cuadro 11).

\section{CONCLUSIONES}

En este trabajo hemos revisado, si bien no exhaustivamente, los principales aspectos metodológicos relacionados con los ECCA cuando se utilizan para realizar proyectos de investigación en el campo de las adicciones. Podemos concluir que estos lineamientos son la base indispensable para llevar a cabo tareas de investigación clínica en esta área con un alto grado de calidad. De esta manera los resultados que se obtengan podrán ser reproducidos por otros centros o bien ser extendidos a otras instancias relativas a los problemas de la salud.

En la Unidad de Ensayos Clínicos de la Subdirección de Investigaciones Clínicas del INPRFM se inició recientemente un proyecto de investigación con una intervención conductual en pacientes con adicciones, para el cual fue necesario llevar a cabo un riguroso entrenamiento metodológico. El aprendizaje y la experiencia derivados del mismo dieron la pauta para poder escribir este artículo. De esta forma, consideramos que su contenido ayudará a establecer los lineamientos mínimos indispensables para futuros estudios.

\section{REFERENCIAS}

1. UNODC. World Drug Report 2012. United Nations publication. Sales No. E.12.XI.1

2. Instituto Nacional de Psiquiatría Ramón de la Fuente Muñiz; Instituto Nacional de Salud Pública; Secretaría de Salud. Encuesta Nacional de Adicciones 2011; México; 2012.

3. Secretaria de Salud. Subsecretaría de Prevención y Promoción de la Salud. Dirección General Adjunta de Epidemiología. Dirección de Investigación Operativa Epidemiológica. Sistema de Vigilancia Epidemiológica de las Adicciones (SISVEA). Informe 2009; México; 2009.

4. World Health Organization. The Global Burden of Disease: 2004 update. United Nations publication.; 2008.

5. Ocampo R., Bojórquez I., Cortés M. Consumo de sustancias y suicidios en México; resultados del Sistema de Vigilancia Epidemiológica de las Adicciones, 1994-2006. Salud Pública Mex; 2009; 51: 306-313.

6. WHO, UNODC. Principles of Drug Dependence Treatment: Discussion Paper. United Nations publication 2008.

7. Degenhardt L., Hall W. Extent of illicit drug use and dependence, and their contribution to the global burden of disease. Lancet 2012; 379:55-70.

8. Strang J., Babor T., Caulkins, J., Fischer B., Foxcroft D., Humphreys K. Drug policy and the public good: evidence for effective interventions. Lancet 2012; 379: 71-83.

9. Friedman LM, Furberg CD, DeMets, DL. Fundamentals of Clinical Trials. 4th. Ed. New York. Springer; 2010. 
10. Lazcano-Ponce E, Salazar-Martínez E, Gutierrez-Castrellón P, AngelesLlerenas A, Hernández-Garduño, A. Viramontes, JL. Ensayos clínicos aleatorizados: variantes, métodos de aleatorización, análisis, consideraciones éticas y regulación. Salud Pública Mex; 2004; 46(6): 559-584.

11. International Conference of Harmonisation of Technical Requirements for Registration of Pharmaceuticals for Human Use. Harmonised Tripartite Guideline: General considerations for Clinical Trials. E8. ICH Harminised Tripartite Guideline; 1997. Obtenido en: www.ich.org.

12. Moher D, Shulz K, Altman D. The CONSORT Statement: revised recommendations for improving the quality of reports of parallel-group randomized trials. JAMA 2001;285(15):1987-1991.

13. McLelland, T. Congratularions on the 10-year anniversary of the National Drug Abuse Treatment Clinical Trial Network - Now, what's new for the coming decade?. Journal of Substance Abuse Tretment; 2010; 38(Suppl 1): S1-S3.

14. Rojas E, Real T, García-Silberman S, Medina-Mora ME. Revisión sistemática sobre tratamiento de adicciones en México. Salud Mental 2011; 34: 351-365.

15. Marín-Navarrete $R$, Benjet $C$, Borges G, Eliosa-Hernández A, NanniAlvarado R, Ayala-Ledesma, M, Fernández-Mondragón J, MedinaMora ME. Comorbilidad de los trastornos por consumo de sustancias con otros trastornos psiquiátricos en centros residenciales de ayudamutua para la atención de las adicciones (CRAMAA). En prensa.

16. Tiet $Q Q$, Mausbach, B. Treatments for patients with dual diagnoses: a review. Alcoholism: Clinical and Experimental Research 2007; 31(4): 513-536.

17. Medina-Mora ME, Borges G, Lara-Muñoz C, Benjet C, Blanco-Jaimes J, Fleiz-Bautista C, Villatoro-Velázquez J, Rojas-Guiot E, ZambranoRuiz J, Casanova-Rodas L, Aguilar-Gaxiola S. Prevalencia de trastornos mentales y uso de servicios: Resultados de la Encuesta Nacional de Epidemiología Psiquiátrica en México. Salud Mental 2003; 26(4):1-16.

18. Carroll K. Dissemination of evidence-based practices: how far we've come, and how much further we've got to go; Addiction 2012; 107: 1031-1033.

19. Lees B, Nitsch D, Wang D, Bakhai A. Patient Selection. En: Wang , Bakhai A (eds). Clinical Trials. A Practical Guide to Design, Analysis, and Reporting. Chicago. Remedica; 2006.

20. Clarke G. Improving the transition from basic efficacy research to effectiveness research in substance abuse treatment. Journal of Substance Abuse Treatment; 1995; 63(5): 718-725.

21. Robbins M, Alonso E, Horigian V, Bachrach K, Burlew K, Carrión I, Hodgkins C, Miller M, Schindler E, VanDeMark , Henderson C, Szapocznik J. Transporting Clinical Research to Community Settings: Designing and Conducting a Multisite Trial of Brief Strategic Family Therapy. Addiction Science \& Clinical Practice; 2010; 5(2): 54-61.

22. Hien D, Cohen L, Campbell A. Methodological Innovation to Increase the Utility and Efficiency of Psychotherapy Research for Patients with Co-ocurring Mental Health and Substance Use Disorders. Professional Psychology Research and Practice; 2009; 40(5): 502-509.

23. Green S. Design of Randomized Trials. Epidemiologic Reviews; 2002; 24(1): 4-11.

24. Carroll K, Onken L. Behavioral Therapies for Drug Abuse. American Journal of Psychiatry; 2005; 162: 1452-1460.

25. Onken L, Blaine J, Battjes, R. Behavioral therapy research: A conceptualization of a process. En: Henngler S, Amentos R (eds), Innovative approaches from difficult to treat populations. Rochester, N.Y: University of Rochester Press; 1997.

26. Rounsaville B, Carroll K, Onken L. A Stage Model of Behavioral Therapies Research: Getting Started and Moving on From Stage 1. Clinical Psychology and Social Practice; 2001; 8:133-142.

27. Knatterud GL. Management and Conduct of Randomized Controlled Trials. Epidemiologic Reviews; 2002; 24(1): 12-25.

28. European Medicines Agency (2006) Statistical Principles for Clinical Trials. Recuperado de http://www.emea.eu.int

29. Guillén A., Carreño A., Canal N. (2006) Fases del análisis estadístico de los datos de un estudio. En Guillén A y Crespo R. (Ed.) Métodos estadísticos para enfermería nefrológica. (pp. 247-260) España: Sociedad Española de Enfermería Nefrológica, SEDEN. Recuperado de http:// www.revistaseden.org/files/17-CAP 17.pdf

30. Lazcano-Ponce E., Salazar-Martínez E., Gutiérrez-Castrellón P., Ángeles-Llerenas A., Hernández-Garduño A., Viramontes J.L. (2009). Ensayos Clínicos Aleatorizados. En Hernández M. (Ed.). Epidemiología: Diseño y análisis de estudios. (1 ${ }^{\mathrm{a}}$ ed., pp.77-119). México: Editorial Médica Panamericana e Instituto Nacional de Salud Pública.

31. Álvarez-Cáceres R. (2005). Ensayos Clínicos: diseño, análisis e interpretación. España: Díaz de Santos.

32. Peduzzi P., Henderson W., Hartigan P., Lavori P. (2002). Analysis of Randomized Controlled Trials. Epidemiol Rev, 1(24), 26-38.

33. Cramer J, Roy A. Burrell A, Fairchild C, Fuldeore M, Ollendorf D, Wong P. Medication Compliance and Persistence: Terminology and Definitions. Value in Health; 2008. 11(1): 44-47.

34. Farmer K. Methods for measuring and monitoring medication adherence in clinical trials and clinical practice. Clinical Therapeutics; 1999; 21(6): 1074-1090.

35. Kazdin AE. Methods of psychotherapy research. En Bongar B, Beutler E (eds.). Comprehensive textbook of psychotherapy: Theory and practice. New York: Oxford University Press.

36. Yeaton, W. H. \& Sechrest, L. (1981). Critical dimensions in the choice and maintenance of successful treatments: Strength, integrity, and effectiveness.(Journal of Consulting and Clinical Psychology, 49, 156167).

37. Carroll K, Martino S, Rousanville B. No Train, No Gain? Clinical Psychology: Science and Practice 2010;17:36-40.

38. Waskow IE. Specification of the technique variable in the NIMH Treatment of Depression Collaborative Research Program. En Williams J, Spitzer R (eds). Psychotherapy research: Where are we and where should we go. New York: Guilford Press;1984.

39. Chambless DL, Hollon SD. Defining empirically supported therapies. Journal of Consulting and Clinical Psychology 1998;66:7-18.

40. Beidas R, Kendall PC. Training therapists in evidence-based practice: A critical review of studies from a systems-contextual perspective. Clinical Psychology: Science and Practice 2010;17: 1-30.

41. Marcus M, Liehr P, Schmitz J, Moeller G, Swank P, Fine M, Cron S, Granmayeh K, Carroll D. Behavioral Therapies Trials: A Case Example. Nurs Res 2007;56(3):210-216.

42. Carroll K, Farentinos C, Ball S, Crits-Christoph P, Libby B, Morgenstern J, Obert J, Polcin D, Woody G. MET meets the real world: design issues and clinical strategies in the Clinical Trials Network. Journal of Substance Abuse Treatment 2002;23:73-80.

43. Miller W, Yahne C, Moyers T, Martínez J, Pirritano M. A Randomized Trial of Methods to Help Clinicians Learn Motivational Interviewing. Journal of Consulting and Clinical Psychology 2004;72:1050-1062.

44. Martino S, Carroll K, Ball S. Teaching, monitoring and evaluating motivational interviewing practice. En Tober G, Raistrick D. (eds). Motivational Dialogue: preparing addiction professionals for motivational interviewing practice. New York: Routledge/Taylor \& Francis Group; 2007.

45. Carpenter KM, Cheng WY, Smith JL, Brooks AC, Amrhein PC, Wain RM, Nunes EV. "Old dogs" and new skills: how clinician characteristics relate to motivational interviewing skills before, during, and after training. J Consult Clin Psychol. 2012;80(4):560-573.

46. Martino S, Ball S, Nich C, Canning-Ball M, Rounsaville B, Carroll K. Teaching community program clinicians motivational interviewing using expert and train-the-trainer strategies. Addiction 2010;106:428-441.

47. Amodeo M, Storti S, Larson, M. Moving Empirically Supported Practices to Addiction Treatment Programs: Recruiting Supervisors to Help in Technology Transfer. Substance Use and Misuse 2010;45:968-982.

48. O'Donovan A, Halford W, Walters B. Towards Best Practice Supervision of Clinical Psychology Trainees. Australian Psychologist 2011;46:101-112. 
49. Sugarman J. Ethics in the Design and Conduct of Clinical Trials. Epidemiologic Reviews; 2002; 24(1): 54-58.

50. Adrian M, Barry S. Physical and mental health problems associated with the use of alcohol and drugs. Substance Use and Misuse; 2003; 38 : 1575-15614.

51. Petry N. Roll J, Runsaville B, Ball S, Stitzer M, Peirce, J, Blaine J, Kirby $\mathrm{K}$, McCarty D, Carroll K. Serious adverse events in randomized psychosocial treatment studies: Safety of Arbitrary Edicts? J Consult Clin Psycol; 2008; 76(6):1076-1082.

52. Horigian V, Robbins M, Dominguez R, Ucha J, Rosa C. Principles for defining adverse events in behavioral intervention research: lessons from a family-focused adolescent drug abuse trial. Clinical Trials; 2010; 7(1): 58-68.

53. McFadden E. Management of Data in Clinical Trials. 2nd. Ed. Hoboken N.J. Wiley Series in Probability and Statistics; 2007.

54. Krishnankutty B, Bellary S, Kumar B, Moodahahu L. Data management in clinical research. Educational Forum; 2012; 44(2): 168-172.

55. Freedman B. Equipoise and the ethics of clinical research. New England Journal of Medicine; 1987; 317(3): 141-154.

56. Ubel P, Silbergleit R. Behavioral equipoise: a way to resolve ethical stalemates in clinical research. The American Journal of Bioethics; 2011; 11(2): 1-8.

57. Weijer C, Shapiro S, Cranley-Glass K, Enkin M. For and Against: Clinical Equipoise and not the uncertainty principle is the moral underpinning of the randomized controlled trial. British Medical Journal; 2000; 321: 756-758.

58. Tribunal Internacional de Núremberg. Código de Núremberg. 1947. Obtenido de: www.bioeticanet.info/documentos/Nuremberg.pdf
59. World Medical Association; WMA Declaration of Geneva. 1948. Obtenido de: http://www.wma.net/en/30publications/10policies/g1/

60. Declaración de Helsinki de la Asociación Médica Internacional. Principios éticos para las investigaciones médicas en seres humanos. Seúl; Asamblea Médica Internacional; 2008. Obtenido en: www.wma.net/ es/30publications/10policies/b3/17c_es.pdf

61. National Commission for the Protection of Human Subjects of Biomedical and Behavioral Research. El Informe Belmont. Principios y Guías éticas para la protección de los sujetos humanos en investigación. EE.UU., 1979. Obtenido en www.bioeticaweb.com/index2. php?option_content\&do_pdf=1\&id=1060.

62. Reglamento de la Ley General de Salud en materia de Investigacion para la Salud. Título Segundo: de los aspectos éticos de la investigación en seres humanos. Capítulo 1: Disposiciones generales. 1983. Obtenido en: http:/www.salud.gob.mx/unidades/cdi/nom/compi/rlgsmis.html

63. International Conference of Harmonisation of Technical Requirements for Registration of Pharmaceuticals for Human Use. ICH Harmonised Tripartite Guideline: Guideline for Good Clinical Practice. E6(R1.; 1996. Obtenido en: www.ich.org.

64. Organización Panamericana de la Salud. Grupo de trabajo en Buenas Prácticas Clínicas. Buenas Prácticas Clínicas: Documento de las Américas. Obtenido en www.paho.org/spanish/ad/ths/ev/BPC-doct-esp.doc

65. Mondragón-Barrios L. Ética en la Investigación Psicosocial. Salud Mental; 2007; 30(6): 25-31

66. Chan A-W, Tetzla JM, Altman DG, et al. SPIRIT 2013 statement: defining standard protocol items for clinical trials. Ann Intern Med 2013; 158 (en prensa).

Artículo sin conflicto de intereses 